Cahiers $d u$ MONDE RUSSE

\section{Cahiers du monde russe}

Russie - Empire russe - Union soviétique et États indépendants

\title{
La révolution russe dans la presse illustrée européenne
}

\section{Anne-Marie Bouchard et Alexis Desgagnés}

\section{OpenEdition}

\section{Journals}

Édition électronique

URL : https://journals.openedition.org/monderusse/9020

DOI : 10.4000/monderusse. 9020

ISSN : $1777-5388$

Éditeur

Éditions de l'EHESS

\section{Édition imprimée}

Date de publication : 15 avril 2007

Pagination : 477-484

ISBN : $978-2-7132-2147-7$

ISSN : $1252-6576$

Référence électronique

Anne-Marie Bouchard et Alexis Desgagnés, "La révolution russe dans la presse illustrée européenne », Cahiers du monde russe [En ligne], 48/2-3 | 2007, mis en ligne le 01 janvier 2007, consulté le 03 septembre 2022. URL : http://journals.openedition.org/monderusse/9020 ; DOI https://doi.org/10.4000/monderusse.9020 
chercher : repérer : avancer

Cet article est disponible en ligne à l'adresse :

http://www.cairn.info/article.php?ID REVUE=CMR\&ID NUMPUBLIE=CMR 482\&ID ARTICLE=CMR 4820477

\title{
La révolution russe dans la presse illust rée européenne
}

\author{
par Anne-Marie BOUCHARD et Alexis DESGAGNÉS
}

\section{Editions de I'EHESS | Cahiers du monde russe}

\author{
$2007 / 2-3-$ Vol 48 \\ ISSN 1252-6576 | ISBN 9782713221477 | pages 477 à 484
}

Pour citer cet article :

-Bouchard A.-M. et Desgagnés A., La révolution russe dans la presse illustrée européenne, Cahiers du monde russe 2007/ 2-3, Vol 48, p. 477-484.

Distribution électronique Cairn pour les Editions de l'EHESS.

(C) Editions de l'EHESS. Tous droits réservés pour tous pays.

La reproduction ou représentation de cet article, notamment par photocopie, n'est autorisée que dans les limites des conditions générales d'utilisation du site ou, le cas échéant, des conditions générales de la licence souscrite par votre établissement. Toute autre reproduction ou représentation, en tout ou partie, sous quelque forme et de quelque manière que ce soit, est interdite sauf accord préalable et écrit de l'éditeur, en dehors des cas prévus par la législation en vigueur en France. Il est précisé que son stockage dans une base de données est également interdit. 


\section{LA RÉVOLUTION RUSSE DANS LA PRESSE ILLUSTRÉE EUROPÉENNE}

Les événements révolutionnaires de 1905 en Russie n'ont cessé de susciter la production d'innombrables images. On évoquera volontiers en guise d'exemples le tableau d'Il'ja Repin intitulé 17 octobre 1905, la fameuse scène de l'escalier Richelieu du Cuirassé Potemkine de Sergej Ejzenštejn, ou encore les illustrations de Boris Lapšin pour une bande dessinée officielle publiée par les Éditions du Progrès au crépuscule de l'histoire soviétique1. Depuis plus d'un siècle, un patrimoine iconographique s'est peu à peu constitué autour du thème de la révolution russe de 1905, composé d'images exposant toutes plus ou moins explicitement le verdict adressé par leur producteur à l'encontre de cette révolution. Il importe de reconnaître et de souligner l'apport considérable de ces représentations au processus d'objectivation historique - voire à la mythification — de la révolution de 1905 en Russie. Puisée au sein de ce patrimoine hétérogène, la présente sélection d'images est appelée à rendre compte de la contribution de la presse illustrée européenne à ce processus ${ }^{2}$.

L'engouement de la presse illustrée européenne pour l'actualité politique russe ne se manifeste pas inopinément avec les événements de janvier 1905. Chaque puissance coloniale européenne ayant des intérêts à défendre en Asie, le conflit qui oppose la Russie et le Japon en Mandchourie domine largement l'actualité politique internationale, et le front russo-japonais constitue déjà un thème iconographique de

1. Evguénia Nikitina et Boris Lapchine, 1905, le prologue, M.: Éditions du Progrès, c1990.

2. Nous avons limité le cadre géographique et temporel de notre étude à certains journaux illustrés publiés en Angleterre, en France et en Allemagne pendant la seule année 1905. Notre sélection s'appuie sur l'examen de titres fréquemment mentionnés dans la littérature secondaire et dont il nous a été possible de consulter la totalité des livraisons pour l'année 1905 : L'Assiette au beurre (Paris), The Graphic (Londres), The Illustrated London News (Londres), Kladderadatsch (Berlin), Le Petit journal (Paris), Punch (Londres), Simplicissimus (Munich), Les Temps nouveaux (Paris) et les suppléments de ces publications. Certains lecteurs remarqueront sans doute ici l'absence du célèbre hebdomadaire parisien l'Illustration. Nous avons exclu ce titre de notre sélection étant donné que la plupart des représentations évoquant l'actualité politique russe qui sont publiées dans ce périodique proviennent de The Illustrated London News. 
prédilection, aussi bien pour les illustrateurs de la presse généraliste à grand tirage que pour les caricaturistes des journaux satiriques. Avec l'année 1905, quelques journaux satiriques spéculent ouvertement sur les destinées du tsarisme. Lorsqu'ils n'ironisent pas sur les perspectives de réformes sociales en Russie (fig. 1), les caricaturistes évoquent tantôt l'inquiétude de Nicolas II quant à la diminution progressive de son prestige dans l'opinion internationale, tantôt son amertume et son désarroi devant un avenir de jour en jour plus imprévisible pour lui. Faute de pouvoir appuyer ces quelques conjectures sur des événements concrets, les caricaturistes sont aussi peu aptes que les illustrateurs de la presse généraliste à anticiper les événements révolutionnaires imminents de Saint-Pétersbourg. La répression sanglante des manifestations du 22/9 janvier à Saint-Pétersbourg - le célèbre Dimanche rouge - aura ceci de particulier qu'elle incitera les médias illustrés à porter leur attention sur la situation politique intérieure de la Russie. Dans l'opinion publique européenne, telle qu'elle se fait jour dans la presse illustrée, ces événements suscitent unanimement l'indignation. La plupart des journaux illustrés étant des hebdomadaires publiés le samedi, c'est dès le 28 janvier qu'apparaissent dans la presse les premières représentations visuelles de la question révolutionnaire en Russie. À partir de cette date, les suppléments et autres numéros spéciaux consacrés à la situation politique russe s'enchaîneront les uns après les autres.

Tout au long de l'année 1905, la couverture de l'actualité révolutionnaire russe par la presse illustrée est assez constante, et ce sont souvent les mêmes événements qui reçoivent généralement l'attention des différents périodiques. Ce fait est garant de la relative cohérence du corpus iconographique constitué par les représentations de la révolution de 1905. Néanmoins, les deux principaux types d'illustrés - généralistes et satiriques - procèdent de visées éditoriales distinctes, voire divergentes, ce qui n'est pas sans incidence sur les modes de représentation privilégiés par ceux-ci. Dans la presse généraliste, l'intérêt porté à l'actualité russe est maintenu par la recension du moindre fait suffisamment spectaculaire pour attiser la curiosité d'un lectorat sensible au pouvoir attractif des images. Le succès commercial de chaque journal dépendant de sa capacité à fournir à son lectorat un contenu relativement crédible, la production des images est conditionnée par une éthique journalistique implicitement revendiquée par la plupart des illustrés généralistes. S'ils n'hésitent pas à envoyer des correspondants sur les lieux de l'action, ces illustrés tablent sur l'objectivité généralement consentie à la photographie pour conférer une certaine valeur documentaire au travail de leurs illustrateurs. La couverture des événements prend donc volontiers la forme de reportages illustrés. La publication de photographies recensant les différents lieux où se déroule l'action sert à échafauder une mise en scène quelque peu didactique devant permettre au lecteur de situer préalablement dans le temps et l'espace l'action évoquée par la représentation graphique (fig. 2 et 3).

Dans les journaux satiriques, la situation politique russe est envisagée de manière plus intermittente, sans pour autant que l'on puisse affirmer que l'intérêt de ces publications pour la question révolutionnaire russe soit moins soutenu que dans la presse généraliste. Considérant le champ politique dans sa totalité, les producteurs de journaux satiriques situent volontiers la question révolutionnaire russe 
dans son rapport avec la situation politique internationale, là où la presse généraliste l'isole pour l'envisager comme une sphère relativement autonome de l'actualité. Dans la mesure où, contrairement aux illustrés généralistes, la plupart des journaux satiriques désavouent toute prétention à l'objectivité journalistique, les représentations satiriques abordant l'actualité révolutionnaire russe sont moins redevables de la contingence de celle-ci. Le mode de production des images étant conditionné par l'ensemble des valeurs idéologiques et des visées stratégiques spécifiant la politique éditoriale de chaque publication satirique, les illustrateurs sont libres de traiter l'actualité révolutionnaire russe par la caricature, voire par le recours à un symbolisme tantôt sibyllin, tantôt parfaitement limpide (fig. 4).

Dès les premières publications évoquant les événements du 22 janvier, le motif du face-à-face opposant soldats et manifestants, motif susceptible de canaliser aisément l'hostilité croissante de l'opinion publique à l'égard du tsarisme, est décliné en maintes représentations (fig.5). Loin de constituer une trouvaille iconographique originale, ce motif consacre une fois de plus l'efficacité d'un dispositif visuel immémorial, consistant en l'évocation synthétique et schématique d'une confrontation entre deux forces antagoniques. D'ordinaire, des légendes accompagnent les représentations du Dimanche rouge, permettant de préciser le sens de celles-ci ou de clarifier la position du périodique vis-à-vis de l'événement représenté. L'usage d'un dispositif visuel relativement simple, mis au service de la diffusion d'un message non moins simple, a indubitablement contribué à imposer rapidement ce motif comme la représentation la plus explicite du climat politique dans la capitale impériale russe et à l'instituer en archétype iconographique par excellence des événements révolutionnaires de 1905. Outre le Dimanche rouge, l'attentat spectaculaire contre le grand-duc Serge (fig. 6), la contagion révolutionnaire en province et en Pologne, l'alliance franco-russe, la mutinerie du cuirassé Potemkine, les pogroms, l'éventuelle formation de la Douma et le manifeste d'octobre sont tour à tour représentés dans la plupart des titres de la presse illustrée, comme autant de symptômes des contradictions structurelles minant la cohésion de l'édifice social russe.

S'il leur importe de signifier l'imminence de l'éclatement de cet édifice en figurant les différents moments où s'opposent les termes de ces contradictions, les périodiques illustrés n'hésitent évidemment pas à identifier les agents de ces oppositions. Le moujik, l'ouvrier, l'étudiant, le terroriste (fig.7), le soldat et le cosaque - types fréquemment rencontrés dans l'iconographie du mouvement révolutionnaire russe - côtoient désormais le marin d'Odessa (fig. 8), ainsi que les différentes personnalités que les circonstances ont fait entrer dans l'histoire du mouvement. Le pope Gapon et l'écrivain Maksim Gor'kij sont ainsi consacrés nouveaux martyrs de la lutte ouvrière, là où le régime tsariste trouve son actualisation dans les figures du grand-duc Vladimir, de Konstantin Pobedonoscev, de Sergej Vitte et, surtout, du tsar Nicolas II (fig. 9). Omniprésent dans l'iconographie de 1905, Nicolas II incarne une institution politique archaïque et vétuste. C'est sur lui que les illustrateurs reportent l'entière responsabilité du chaos qui sévit dans l'Empire. Tantôt préoccupé, désespéré, furieux ou sanguinaire, tantôt indifférent, 
incompétent, inconscient ou parfaitement niais, Nicolas est présenté dans la plupart des illustrés comme un souverain médiocre, incapable de gérer convenablement les affaires politiques et entaché par le sang de son peuple (fig. 10 et 11).

Les spectres de la Révolution française font fréquemment leur apparition dans la presse satirique. Dès avant le Dimanche rouge, les caricaturistes les plus lucides formulent déjà l'hypothèse d'une répétition historique du 21 janvier 1793 pour signifier ce qui, en Russie, n'est encore qu'un présage (fig. 12). Ainsi, voit-on fréquemment le fantôme de Louis XVI s'entretenir avec Nicolas II, le revenant allant même jusqu'à se faire le défenseur du compromis constitutionnel devant sauver la tête du tsar (fig. 13). En plus de signifier l'impossibilité pour Nicolas II d'échapper à son sombre destin, les références à la Révolution française dans la presse satirique visent à situer historiquement les événements russes dans la continuité des révolutions européennes du XIXe siècle et à conférer aux actions du mouvement révolutionnaire russe une certaine légitimité, malgré les dérives possibles. Si les sympathies des périodiques satiriques pour la cause révolutionnaire sont inversement proportionnelles à leur hostilité envers Nicolas, elles sont considérablement atténuées dans les illustrés généralistes. Bien que manifestement sensible aux revendications du mouvement ouvrier russe pendant les premiers mois de 1905, la presse généraliste multiplie au fil de l'année la parution d'illustrations montrant des scènes de saccages, d'actes de vandalisme et de jacqueries, perpétrés par les masses populaires en général, et par les anarchistes et les communistes en particulier (fig. 14 et 15). La crainte d'assister à l'avènement d'un « règne de la Terreur » semble attiser la suspicion de ce type de publication quant à la légitimité politique de débordements populaires portant potentiellement atteinte à la propriété privée et à l'État. Alors que la presse généraliste illustrée réduit souvent les aspirations révolutionnaires des Russes au caractère destructeur des manifestations les plus spectaculaires, la presse satirique conçoit plutôt cet élan destructeur comme le premier temps d'une transformation du monde, certes violente, mais néanmoins nécessaire. Très critiques des concessions politiques trop facilement arrachées au tsar par le mouvement ouvrier russe, les caricaturistes semblent craindre la possible stagnation de l'impulsion révolutionnaire. C'est pourquoi, aux lendemains de la proclamation du manifeste d'octobre, les représentations dénonçant tout compromis constitutionnel foisonnent dans la presse satirique. Dans l'esprit des caricaturistes, même quand la révolution se fait destructrice, elle anticipe nécessairement la réalisation inéluctable d'un monde nouveau, qui ne pourra être édifié que sur les ruines du tsarisme (fig. 16).

Outre l'intérêt que génère sa contemporanéité avec les événements révolutionnaires de 1905, la contribution la plus substantielle de la presse illustrée au patrimoine iconographique de la révolution russe de 1905 nous semble résider dans le fait que, dans les représentations qu'elle produit, la révolution n'est pas tant envisagée comme une entité historique finie que comme une potentialité. Certes, l'immédiateté avec laquelle les périodiques illustrés ont réagi à l'actualité politique russe a limité considérablement leur capacité à inscrire leur analyse de celle-ci dans la longue durée, induisant ainsi une tendance à polariser fortement les enjeux politi- 
ques représentés. Néanmoins, en opérant les choix qu'imposait la production de son contenu iconographique, la presse illustrée a contribué à consolider la signification historique des événements révolutionnaires de 1905, peut-être même avant qu'elle n'eût été pleinement comprise. C'est cette contribution que nous avons ici souhaité présenter sommairement.

Au-delà des limites temporelles et géographiques que nous avons assignées à notre présentation, l'examen de cette contribution mériterait évidemment d'être complété par l'exposition des liens existant entre la presse illustrée européenne et la presse illustrée russe. En Russie, la particularité des conditions de production des représentations associées au thème de la révolution russe de 1905 différencie considérablement la nature de ces représentations de celle des images constituant le corpus discuté dans le présent article. Toutefois, certains indices nous permettent de constater l'existence d'une réception contemporaine de la propagande illustrée russe dans la presse européenne ${ }^{3}$. La documentation des réseaux de diffusion de cette propagande nous permettrait de poser la question des rapports d'influences réciproques entre ces deux types de production iconographique. Par ailleurs, il serait également pertinent d'examiner dans quelle mesure la réception et la représentation des événements révolutionnaires de 1905 dans la presse illustrée japonaise furent conditionnées par le conflit mandchou.

Département d'histoire de l'art et d'études cinématographiques

Université de Montréal

alexis.desgagnes-tremblay@umontreal.ca

anne-marie.bouchard.4@umontreal.ca

\section{Bibliographie}

L'Assiette au beurre, Paris, [s.n.], 1901-1912.

The Graphic. An Illustrated Weekly Newspaper, Londres, [s.n.], 1869-1932.

The Illustrated London News, Londres, Illustrated London News \& Sketch Ltd, 1842-.

Kladderaddatsch, Humoristisch-satirisches Wochenblatt, Berlin, A. Hofmann \& Co Verlag, 1848-1944.

Le Petit journal, Paris, [s.n.], 1863-1944

Punch, or the London Charivari, Londres, Punch Publications Ltd, 1841-.

Le Rire, Journal humoristique paraissant le samedi, Paris, F. Juven, 1894-1959.

Simplicissimus. Illustrierte Wochenschrift, Munich/Stuttgart, A. Langen Verlag, 1896-1944.

Les Temps nouveaux, Paris, [s.n.], 1895-1921.

3. Une caricature russe est reproduite dans la livraison du 30 décembre 1905 de l'hebdomadaire anglais The Graphic. 
ALLEN, Ann Taylor, Satire and Society in Wilhelmine Germany : "Kladderadatsch" and "Simplicissimus", 1890-1914, Lexington : University press of Kentucky, 1984.

BACOT, Jean-Pierre, La presse illustrée au XIX $X^{\mathrm{e}}$ siècle : une histoire oubliée, Limoges : PULIM, 2005.

DARDEL, Aline, Les Temps nouveaux, 1895-1914. Un hebdomadaire anarchiste et la propagande par l'image, Paris : Les dossiers du Musée d'Orsay, 1987.

DIXMIER, Elisabeth et DIXMIER, Michel, L'Assiette au beurre : revue satirique illustrée, 1901-1912, Paris : F. Maspéro, 1974.

NIKITINA , Evguénia et LAPCHINE, Boris, 1905, le prologue, Moscou : Éditions du Progrès, 1990.

STERN, Leo, ed., Despotie in der Karikatur. Die russische Revolution 1905-1907 im Spiegel der deutschen politischen Karikatur, Berlin: Akademie-Verlag, 1967.

\section{Figures}

1 Anonyme, « Der Stillstand der Reformen in Rußland», Kladderadatsch, vol. 58, n 3 (15 janvier 1905), p. 12. Légende : «Wenn eine Staatsmühle oberschlächtig und unterschlächtig zugleich gebaut ist, darf man nicht beide schleifen zu gleicher Zeit aufmachen ».

2 «Scenes of Strikes and Massacre: the Mise-en-scene and Dramatis Personæ », The Illustrated London News, vol. 126, n³432 (28 janvier 1905), p. 111.

3 «The St. Petersburg Massacre from Sketches by our Special Artist », The Graphic, vol. 71, n 1836 (supplément du 4 février 1905), p. 1.

4 Anonyme, «Der russische Koloß», Kladderadatsch, vol. 58, n 11 (12 mars 1905), p. 44. Légende : «Auf europäischer wie auf asiatischer Seite steht er mit einem Fuß im Feuer und ist vergeblich bemüht es zu löschen ».

5 H. W. Koekkoek, «The Cossacks' Way with the Crowd: Charging the Strikers with loaded Whips on January 22 », The Illustrated London News, vol. 126, n 3432 (28 janvier 1905), p. 112. Légende: "First blood in the Revolution : repulsing the strikers with sword, whip, and gunshot opposite the admiralty building, St. Petersburg ».

6 Anonyme, «Attentat révolutionnaire à Moscou », Le Petit Journal, supplément illustré, vol. 16, n 746 (5 mars 1905), p. 73. Légende : «Le Grand-Duc Serge, oncle du Tsar, tué par l'explosion d'une bombe ».

7 Thomas Theodor Heine, «Russische Bombentechnik», Beiblatt des Simplicissimus, vol. 9, n 50 (7 mars 1905), p. 499. Légende : «Alle Höfe Europas trauern um Sergius, ich aber trauere um den armen Kutscher. Jetzt will ich eine Bombenfüllung erfinden, die auf Kutscher nicht wirkt ».

8 René-Georges Hermann-Paul, «Les marins d'Odessa », Les Temps nouveaux, Supplément littéraire illustré, vol. $5, \mathrm{n}^{\circ} 10 / 7$ (8 juillet 1905), p. 52-53. Légende : « Notre ennemi, c'est notre maître !»

9 «In the Uniform of the Butchers : the Tsar as a Cossack», The London Illustrated News, vol. 126, $\mathrm{n}^{\circ} 3432$ (28 janvier 1905), p. 105. Légende : « Here the tsar is seen in a Cossack's full equipment, down even to the "naghaika", or short-handed whip, wich has frequently been used for the repression of rioters. On January 22 the Cossacks used their whips against a deputation of strikers, which had been sent to the barracks to protest against the violence of the military ». 
10 Demetrios Galanis, «Le Tzar rouge », L'Assiette au beurre, n 201 (4 février 1905), p. 3321. Légende : "Peuple russe, tremblant et morne, tu chemines; Serf à SaintPétersbourg, ou forçat dans les mines; Le pôle est pour ton maître un cachot vaste et noir; Russie et Sibérie, o czar! tyran! vampire !; Ce sont les deux moitiés de ton funeste empire ; L'une est l'Oppression, l'autre est le Désespoir ! (Victor Hugo, Les Châtiments) ».

11 Olaf Gulbransson, «Der blinde Zar», Simplicissimus, illustrierte Wochenschrift, vol. $9, n^{\circ} 48$ (21 février 1905), p. 471.

12 Thomas Theodor Heine, «Wandgemälde für das Petersburger Schloß », Simplicissimus, illustrierte Wochenschrift, vol.9, n 43 (17 janvier 1905), p. 421. Légende : «Lange Zeit konnte sich Ludwig XVI nicht entschließen, seinem Volke eine Verfassung zu geben. Als man ihn endlich dazu zwang, mußte er sein „, Ja “ so heftig nicken, daß ihm der Kopf von den Schultern flog ».

13 Linley Sambourne, «The Eleventh Hour », Punch, or the London Charivari, vol. 129 (15 novembre 1905), p. 353. Légende : « Side with your people, Sire, while there is yet time. I was too late! »

14 Anonyme, «La jacquerie dans les provinces russes », Le Petit Journal, supplément illustré, vol. 16, n 750 (2 avril 1905), p. 112. Légende : «Invasion d'un château par des paysans russes révoltés ».

15 F. de Haenen, «Under the Red Flag: A Street in Moscow in the Hands of a Mob of Strikers », The Graphic, vol.71, n 1876 (supplément du 11 novembre 1905), p. 1. Légende : "Our Correspondent writes - "A vast multitude of strikers and rioters passed down the Pokrovka Street, smashing windows and destroying the street lamps. The red revolutionary flag is now becoming a commun sight in the city, and whenever it makes its appearance ther is destruction and pillage." »

16 Demetrios Galanis, «Demain... Sur les ruines du Palais d'hiver», L'Assiette au beurre, n $^{\circ} 201$ (4 février 1905), p. 3336. Légende : «Avenir ! Avenir! Voici que tout s'écroule ! Les pâles rois ont fui, la mer vient, le flot roule. Peuples ! Le clairon sonne aux quatre coins du ciel. (Victor Hugo, Les Châtiments)». 